\title{
Differences between foreign exchange rate regimes: the view from the tails
}

\author{
Kees G. KoediJk \\ Rijksuniversiteit Limburg, PO Box 616, 6200 MD Maastricht, The Netherlands \\ Philip A. STORK \\ Erasmus Universiteit Rotterdam, PO Box 1738,3000 DR Rotterdam, The Netherlands \\ AND \\ Casper G. De Vries \\ Katholieke Universiteit Leuven and Erasmus Universiteit Rotterdam, PO Box \\ 1738, 3000 DR Rotterdam, The Netherlands
}

\begin{abstract}
In the literature on the empirical unconditional distribution of foreign exchange rate returns there is indication that the type of distribution function is related to the form of exchange rate regime. The analysis has been hampered by the nonnestedness of alternative distribution models. The paper investigates the issue by means of extremal analysis which allows for a unified treatment. In particular, we try to sort out whether apparent distributional differences are due to differences in techniques or in regimes. (JEL F31, F33)
\end{abstract}

The research on the unconditional distribution of foreign exchange rate returns is organized around the one important stylized fact that the empirical distribution has fat tails. Therefore distributions like the Student- $t$ and stable, for which not all moments are defined, are used in modeling exchange rate returns, while the normal distribution, for example, is not employed. ${ }^{1}$ The conclusions from the few papers that have estimated these models across different exchange rate regimes are as follows. Westerfield (1977) and Rana (1984) estimated the stable model on pre and post Bretton Woods data, i.e., for a fixed and floating regime, and found a generally higher characteristic exponent $\beta$ for the latter period. ${ }^{2} \mathrm{~A}$ rise in the characteristic exponent implies a decline of probability mass in the tails. Using Westerfield's data Rogalski and Vinso (1978) estimated the Student- $t$ model and found a slightly higher degrees of freedom parameter $v$ for the floating period,

\footnotetext{
*We are grateful to Vedat Akgiray and Geofirey Booth for providing us with their data set for Latin America, and to Folkert Pieterse for collecting additional data. We benefited from presentations at the Econometric Society European Winter Meetings in Poland and C.O.R.E., Louvain-la-Neuve. The comments and suggestions made by two anonymous referees and Eduard Bomhoff are gratefully acknowledged.
} 
thus indicating somewhat thinner tails for the float. In summary, both types of studies corroborate the theoretical presumption that a float smoothens the fluctuations in exchange rates vis-à-vis a fixed parity system. Interestingly, Rogalski and Vinso by using the likelihood ratio of the Student versus stable model also find that the stable model has the higher posterior odds (using the Bayesian interpretation of the likelihood ratio) for the period of fixed rates, while the Student model dominates for the float. Hence, a regime change may not only affect a model's parameters, but the entire model, i.e., inducing a switch from the stable to the Student $t$ model. The problem with these alternative data-generating mechanisms is that they are nonnested in the parameter space. This impairs the usefulness of the likelihood ratio criterion as its asymptotic distribution is unknown. Hence one cannot determine whether the regime switch had any significant effects in terms of model changes.

In this paper we employ extremal analysis in order to overcome this difficulty, and use the econometric techniques introduced by Koedijk et al. (1990). ${ }^{3}$ As was indicated above, the organizing principle is the fatness in the tails of the distribution. Extremal analysis investigates the distribution of the maximum (minimum) in large samples, thereby determining the shape of the tails of a distribution. In fact, the limit law for the maximum is characterized by, the socalled tail index $\alpha$, which happens to be one to one with the number of moments that exist. Thus $\alpha$ is a good indicator for the thickness of the tails. The important point to note is that the alternatives like the stable and Student- $t$ model are nested within the limit law for extremes, as the characteristic exponent $\beta<2$ corresponds to values $\alpha<2$, while the degrees of freedom $v \geqslant 2$ equal $\alpha \geqslant 2$. The idea is then to estimate this tail index directly, and to use the known distribution of the tail estimator in order to test for regime switches affecting the type of distribution. The gain of this procedure is that one can nest and test for different tail sizes. The loss consists of information about the center characteristics of the distribution. Given the predominance of outliers in the return series, however, one may benefit from this tradeoff for studying issues like exchange rate volatility.

Interestingly, in a recent issue of this journal, Akgiray et al. (1988) studied the distribution of black market exchange rate returns using extremal analysis as one of their techniques. On the basis of the maximum likelihood procedure Akgiray et al. find for 12 Latin American countries $\alpha$ values which range between 1/2 and 7. This diversity is somewhat disturbing as studies that estimate a specific distribution typically imply $\alpha$ values for different currencies which do not vary much, see, e.g., Boothe and Glassman (1987). The diversity may be due to the specific regime. ${ }^{4}$ However, the reported confidence intervals are also rather large. In fact, the maximum likelihood procedure is not the most efficient method as the Cramér-Rao bound does not apply. Recently simple nonparametric estimators which are more efficient have become available. (see the review by Hols and De Vries, 1991). Therefore, we propose to use these new estimators to estimate the tail index of exchange rate returns under alternative regimes.

To summarize, in this paper we attempt to overcome the maintained hypothesis problem by following the device of Akgiray et al. and employing extremal analysis. The distribution of the extremes nests the alternative models in a natural way. To improve upon the maximum, likelihood procedure, we employ a more efficient nonparametric approach described below. In order to provide a comparison of techniques we employ the same data set for the analysis of black market returns 
as Akgiray et al. The nonparametric estimator is then applied to foreign exchange rate returns from five different regimes: Bretton Woods, the Snake, the free float, the EMS, and various black markets. In this way we are able to sort out unconditionally how regime switches affect the distribution of the returns and the behavior of 'outliers' in particular.

\section{Theory and simulations}

For the purpose of this paper it suffices to provide a brief summary of the theory of extremes. The interested reader is referred to Hols and De Vries (1991) for theoretical details. Consider a stationary sequence $X_{1}, X_{2}, \ldots, X_{n}$ of independent and identically distributed (i.i.d.) random variables with a distribution function $F$ (d.f. $F$ ). Suppose one is interested in the probability that the maximum

$$
M_{n}=\max \left(X_{1}, X_{2}, \ldots, X_{n}\right)
$$

of the first $n$ random variables is below a certain level $x$. As is well known, this probability is given by

$$
P\left\{M_{n} \leqslant x\right\}=F^{n}(x) .
$$

Extreme value theory studies the limiting distribution of the order statistic $M_{n}$ appropriately scaled. That is, one is interested under which conditions there exist suitable normalizing constants $a_{n}>0, b_{n}$, such that:

$$
P\left\{a_{n}\left(M_{n}-b_{n}\right) \leqslant x\right\} \rightarrow G(x),
$$

where $G(x)$ is an extreme value distribution. If $F(x)$ is fat tailed, i.e., $F$ is regularly varying at infinity, then $b_{n}=0$, and for $y=x / a_{n}$ :

$$
G(y)=e^{-t y^{x}},
$$

where $x$ is the tail index, $x>0$, and $\tau$ is the scaling parameter which is a function of the marginal distribution of the $X_{i}$ and the possible dependency between the $X_{i}$ s. Formal results for the i.i.d. and dependent case are given in Leadbetter $e t$ al. (1983). Values for $x$ for specific d.f. $F(x)$ and processes are as follows. ${ }^{5}$ The Student $t$ model has the tail index $\alpha$ equal to its degrees of freedom $v$, see, e.g., Mood et al. (1974, p. 262). The fat tailed stable models have $\alpha$ equal to the characteristic exponent $0<\beta<2$. The ARCH(1) model $Y_{t}=X_{t}\left(\beta+\lambda Y_{t-1}^{2}\right)^{1 / 2}$ has a tail index which is defined implicitly by the following equation $\Gamma((\alpha+1) / 2)=\pi^{1 / 2}(2 \lambda)^{-\alpha / 2}$, and where the $X_{t}$ are i.i.d. $N(0,1)$ (see De Haan $e t$ al., 1989). Thus, the stable model implies $0<x<2$, while the Student- $t$ and ARCH model allow for $\alpha \geqslant 2$.

How to estimate the tail index $\alpha$ ? For notational convenience let $\gamma=1 / \alpha$ denote the inverse tail index. As is shown in Smith (1987), $\gamma$ can be estimated consistently by maximum likelihood (m.l.) and this estimator $\hat{\gamma}_{m l}$ is asymptotically normal distributed: ${ }^{6}$

$$
\left(\hat{\gamma}_{m l}-\gamma\right) \sqrt{ } m \sim N\left(0,(1+\gamma)^{2}\right),
$$

where $m$ is the number of highest observations from a sample of size $n>m$. Next, consider the nonparametric estimator proposed by Hill (1975). Define $X_{(1)} \leqslant X_{(2)} \leqslant \cdots \leqslant X_{(n)}$ as the ascending order statistics from a sample $X_{1}, \ldots, X_{n}$. 
The Hill estimator reads

$$
\hat{\gamma}_{h}=\frac{1}{m} \sum_{i=1}^{m}\left[\log X_{(n+1-i)} / X_{(n-m)}\right] .
$$

Goldie and Smith (1987) have shown that asymptotically

$$
\left(\hat{\gamma}_{h}-\gamma\right) \sqrt{ } m \sim N\left(0, \gamma^{2}\right) .
$$

Remark, if the distribution $F(x)$ is symmetric, both tails have the same index. Moreover, asymptotically the maxima and minima are independent, c.f. Leadbetter et al. (1983). It then pays to pool the highest and lowest observations by taking the absolute value and then ordering from low to high.

From $\langle 5\rangle$ and $\langle 7\rangle$ it is immediate that for given $m, \hat{\gamma}_{h}$ is asymptotically more efficient than $\hat{\gamma}_{m l}$ by a factor $(1+\alpha)^{-2}$. The importance of this result can be motivated by the following example given in Dumouchel (1983). In his study Dumouchel investigates the relative efficiency of the Smith m.l. procedure with the m.l. procedure for estimating all the parameters of a d.f., if it were known that the true d.f. is stable. Evidently, the former estimator will be less efficient than the latter, hecause the latter estimator exploits the extra a priori information. For $\beta=\alpha=1.5$, Dumouchel $(1983$, p. 1028) concludes: 'Thus the efficiency of the robust procedure is only 0.034 , a large price to pay for robustness.' Continuing Dumouchel's example and using our nonparametric technique shows, however, that the nonparametric $\hat{\gamma}_{h}$ is 4.75 times more efficient than Smith's $\hat{\gamma}_{m l}$, but only 0.16 times as efficient as the m.l. procedure for estimating $F$ if the d.f. is known to be stable. The gain from using the nonparametric technique is thus quite considerable. One should also bear in mind that it is usually not known whether the true d.f. $F(x)$ is stable or not. In this vein, Dumouchel writes: 'Alternative strategies which fit $t$-distributions or other families of long tailed distribution to all of the data are beside the point. Presumably, any method which uses the central observations to help make inferences about tail behavior is somewhat nonrobust.'

The intuition behind the dominance of $\hat{\gamma}_{h}$ over $\hat{\gamma}_{m l}$ is as follows. The m.l. estimator is based on the hypothesis that the limit law is the correct model, whereas it holds only in an approximate sense for finite sample sizes (and $F(x)$ unequal to $G(x)$ ). Therefore the m.l. estimator does not attain the Cramér-Rao bound, and more efficient estimators may exist. In order to demonstrate the relative efficiency of the two estimators, we conducted a simulation study. Before presenting the results of this study, however, we discuss a second motive for running the experiments.

A problem associated with all extreme value estimation techniques is that they require one to select a number $m$ of highest order statistics. While $m(n)$ has to go to infinity at a lower rate than the sample size $n$, it is not known how to choose $m$ optimally. In the numerical example discussed above, Dumouchel suggested the use of $m=n / 10$ if one samples from one of the tails. Our simulations below show that this procedure is suboptimal in general. We exploited the asymptotic normality of the estimators to select an optimal $m$ on the basis of the MSE criterion. In order to achieve this, we ran a Monte Carlo experiment by drawing from different d.f.s $F(x)$. For each distribution the tail indexes were calculated for different $m$-levels. Replications were used to compute the associated MSE and Bias squared. The optimal $m$-levels for given d.f. and estimation 
TABLF 1. MSE, Bias squared and $m$-levels.*

Sample size $n=119,500$ replications

\begin{tabular}{cccccc}
\hline Distribution & \multicolumn{2}{c}{$\alpha=1$} & \multicolumn{3}{c}{$\alpha=3$} \\
\hline Estimator & $\hat{\alpha}_{m l}$ & $\hat{\alpha}_{h}$ & $\hat{\alpha}_{m l}$ & $\hat{\alpha}_{h}$ \\
\hline$m$ & & & & & \\
6 & $0.58(0.15)$ & $0.20(0.00)$ & $0.13(0.02)$ & $0.04(0.01)$ \\
8 & $0.46(0.07)$ & $0.13(0.00)$ & $0.11(0.01)$ & $0.03^{*}(0.01)$ \\
12 & $0.37(0.05)$ & $0.09(0.00)$ & $0.09(0.02)$ & $0.04(0.02)$ \\
16 & $0.26(0.04)$ & $0.08(0.00)$ & $0.08(0.01)$ & $0.05(0.03)$ \\
18 & $0.24(0.03)$ & $0.06^{*}(0.00)$ & $0.08(0.02)$ & $0.05(0.04)$ \\
22 & $0.21(0.02)$ & $0.06(0.01)$ & $0.06(0.02)$ & $0.08(0.07)$ \\
26 & $0.20(0.01)$ & $0.07(0.02)$ & $0.06(0.03)$ & $0.12(0.10)$ \\
28 & $0.19(0.03)$ & $0.06(0.02)$ & $0.06(0.03)$ & $0.14(0.13)$ \\
32 & $0.16(0.03)$ & $0.08(0.04)$ & $0.06^{*}(0.03)$ & $0.21(0.19)$ \\
36 & $0.14(0.03)$ & $0.12(0.08)$ & $0.06(0.03)$ & $0.30(0.30)$ \\
50 & $0.13(0.05)$ & $1.74(1.45)$ & $0.06(0.04)$ & $1.30(0.89)$ \\
\hline
\end{tabular}

*The first column reports the number of order statistics $m$ taken into account. The other columns record the MSE and Bias squared between brackets. Stars refer to the minimum MSE. Note, MSE and Bias are computed for $\gamma=1 / x$.

technique are recorded in Table 1 together with the associated MSE and Bias squared. The sample size of Table 1 corresponds to the sample size of Akgiray et al. ${ }^{7}$ Two distributions were used in the simulations: the Cauchy, as it is a member of the stable distributions with $\alpha=\beta=1$; and the Student $t$ with 3 degrees of freedom, i.e., $\alpha=v=3$.

From Table 1 it is immediate that the optimal $m$-level varies considerably with the type of distribution function $F(x)$. As the Cauchy distribution has more probability mass in the tails than the Student $t$ with $v=3$, a larger number of extreme realizations can be used for estimation than for the Student model. The number of order statistics needed for the m.l. approach, though, is quite large (for $x=1$ the lowest MSE was obtained at the highest $m$-value used in the experiment; Akgiray et al. recommend setting $m=18$ ). In order to check the usefulness of extreme value analysis, the theoretically predicted MSE levels can be compared with the simulated outcomes at the $m$-levels where the MSE is minimal. For example, when $\alpha=3$ and $m=32$ the theoretical MSE for $\hat{\gamma}_{m l}$ is 0.06 and for $m=8$ the value for $\hat{\gamma}_{h}$ is 0.01 .

It is apparent that both theoretical values are close to the experimental MSE values. Thus extreme value analysis seems a useful tool, but one has to exercise care in selecting an appropriate $m$-level when applying the estimators. In particular, the m.l. estimator requires much higher $m$-levels. As the MSEs at the optimal $m$-levels are quite close to the theoretically predicted MSE, it also follows from the simulations that the Hill estimator dominates the m.l. estimator. Continuing the above example, i.e., $\alpha=3, n=119, \hat{\gamma}_{m l}$ has an experimental MSE of 0.06 , while the MSE for $\hat{\gamma}_{h}$ is 0.03 respectively, thus the relative efficiency is $0.06 / 0.03=2$. Theoretically one expects an asymptotic relative efficiency of $(1+\alpha)^{2} *\left(m_{h} / m_{m l}\right)=16 *(8 / 32)=4$. Clearly, the Hill estimator dominates, but by less than would be expected on the basis of the asymptotic difference. Using a 
much larger sample of $n=6000$ (see note 7) the same computations yielded an asymptotic difference of $16(75 / 400)=3$, while the simulations gave $0.0063 / 0.0023=2.74$, which is quite close to the theoretical value. Henceforth we propose to use the Hill estimator instead of the m.l. procedure.

Before we turn to the empirical application, we present one more theoretical result. An obvious and interesting question is whether the $\alpha$ values for the different regimes will be significantly different from each other. Due to the independence of the samples and asymptotic normality of the estimator $\hat{\alpha}_{h}$. we are able to test for this by means of the following statistic $Q$ :

$$
Q=\left[\frac{\alpha_{1}}{\hat{\alpha}_{1}}-1\right]^{2} m_{1}+\left[\frac{\alpha^{2}}{\hat{\alpha}_{2}}-1\right]^{2} m_{2},
$$

where $\alpha=1 / \gamma$ and $m$ are as in $\langle 7\rangle$, and the subindexes refer to two independent samples. It is easy to see that $Q$ is asymptotically $\chi^{2}(2)$ distributed.

\section{Empirical results}

In this section we apply the above methodology to estimate the tail index of exchange rate returns for different regimes. To enhance comparability, we start with results for the black market regime. In Table 2 we have applied the nonparametric estimation procedure to the data of Akgiray et al. (1988), as well as to an extended sample. ${ }^{8}$ Because the extreme valuc method rclies on there being a sufficient number of tail observations, increasing the relatively small sample size of 119 used by Akgiray et al. may potentially lead to improved estimates. The restrictiveness of the stable model vis-à-vis the entire class of fat tailed distributions is clear from the contrast between the first column and the others. The characteristic exponent $\beta$ hovers around 1 while some of the tail indexes are above 2 , thus pointing to a finite variance. The second column records Akgiray et al.'s application of the Smith $\gamma_{m l}$ procedure for estimating the tail index. ${ }^{9}$ Not only is there a wide diversity in $\alpha$ values, the confidence intervals are also very large, leaving much uncertainty. A negatively valued right end point of the confidence interval has to be interpreted as follows. In general the inverse tail index $\gamma$ can be positive, negative, or zero. If $\gamma<0$, the domain of the d.f. $F(x)$ is bounded from above, and if $\gamma=0$ the d.f. $F(x)$ has thin tails like the normal distribution. Therefore, a negative right end point for $\alpha$ indicates that the hypothesis of thin tailed distributions, $\alpha=\infty$, or bounded random variables, $\alpha<0$, cannot be rejected. All other evidence, however, shows that these latter two alternatives are not plausible models for nominal exchange rate returns.

The third and fourth column record tail index values that we found on the basis of the more efficient Hill estimator. ${ }^{10}$ The second and third column are based on the same sample, but the third column has mostly tighter confidence intervals and all point estimates hover around 2. For the extended sample the point estimates are somewhat lower, and in some cases significantly below 2 , thereby supporting the stable hypothesis. To conclude, theory, simulations, and empirical applications show that the nonparametric approach to extreme value problems is a relatively efficient technique which can be used to estimate and test hypotheses about the tail index of exchange rate returns.

Given this conclusion, we broaden our scope and investigate regimes other than the black market. Table 3 provides tail index estimates for three different 
TABLF 2. Black market tail indexes.*

\begin{tabular}{|c|c|c|c|c|c|}
\hline Country & $\begin{array}{c}\text { Characteristic } \\
\text { exponent } \\
\hat{\beta}\end{array}$ & $\hat{\alpha}_{m l}$ & $\begin{array}{l}\text { Tail inde } \\
\hat{\alpha}_{h}\end{array}$ & $\hat{\alpha}_{h}$ & $n(\mathrm{~m})$ \\
\hline Argentina & 1.00 & $\begin{array}{c}1.24 \\
(0.65,14.20)\end{array}$ & $\begin{array}{c}2.12 \\
(0.65,3.59)\end{array}$ & $\begin{array}{c}1.83 \\
(1.17,2.48)\end{array}$ & $\begin{array}{l}443 \\
(30)\end{array}$ \\
\hline Bolivia & 0.57 & $\begin{array}{c}0.97 \\
(0.54,4.55)\end{array}$ & $\begin{array}{c}1.85 \\
(0.57,3.13)\end{array}$ & $\begin{array}{c}2.26 \\
(1.45,4.36)\end{array}$ & $\begin{array}{l}431 \\
(29)\end{array}$ \\
\hline Brazil & 1.49 & $\begin{array}{c}6.90 \\
(1.90,-4.25)\end{array}$ & $\begin{array}{c}3.72 \\
(1.14,6.31)\end{array}$ & $\begin{array}{c}3.21 \\
(2.06,4.36)\end{array}$ & $\begin{array}{l}443 \\
(30)\end{array}$ \\
\hline Chile & 0.84 & $\begin{array}{c}0.58 \\
(0.35,1.61)\end{array}$ & $\begin{array}{c}4.34 \\
(1.33,7.34)\end{array}$ & $\begin{array}{c}2.34 \\
(1.51,3.18)\end{array}$ & $\begin{array}{l}431 \\
(29)\end{array}$ \\
\hline Colombia & 1.29 & $\begin{array}{c}6.45 \\
(1.89,-4.56)\end{array}$ & $\begin{array}{c}1.46 \\
(0.44 .2 .47)\end{array}$ & $\begin{array}{c}1.73 \\
(1.06,2.39)\end{array}$ & $\begin{array}{l}383 \\
(26)\end{array}$ \\
\hline Costa Rica & 0.78 & $\begin{array}{c}2.20 \\
(1.07,-41.73)\end{array}$ & $\begin{array}{c}1.76 \\
(0.54,2.98)\end{array}$ & $\begin{array}{c}1.25 \\
(0.80,1.70)\end{array}$ & $\begin{array}{l}431 \\
(29)\end{array}$ \\
\hline Ecuador & 0.97 & $\begin{array}{c}2.57 \\
(1.17,-12.68)\end{array}$ & $\begin{array}{c}2.02 \\
(0.62,3.42)\end{array}$ & $\begin{array}{c}1.55 \\
(1.00,2.11)\end{array}$ & $\begin{array}{l}443 \\
(30)\end{array}$ \\
\hline El Salvador & 0.74 & $\begin{array}{c}1.52 \\
(0.70,-8.29)\end{array}$ & $\begin{array}{c}1.87 \\
(0.57,3.17)\end{array}$ & $\begin{array}{c}1.27 \\
(0.68,1.86)\end{array}$ & $\begin{array}{l}275 \\
(18)\end{array}$ \\
\hline Nicaragua & 0.52 & $\begin{array}{c}1.58 \\
(0.77,-29.60)\end{array}$ & $\begin{array}{c}2.07 \\
(0.63,3.50)\end{array}$ & $\begin{array}{c}1.38 \\
(0.88,1.87)\end{array}$ & $\begin{array}{l}443 \\
(30)\end{array}$ \\
\hline Paraguay & 1.20 & $\begin{array}{c}0.61 \\
(0.37,1.78)\end{array}$ & $\begin{array}{c}1.31 \\
(0.40,2.21)\end{array}$ & $\begin{array}{c}1.42 \\
(0.87,1.96)\end{array}$ & $\begin{array}{l}383 \\
(26)\end{array}$ \\
\hline Peru & 1.82 & $\begin{array}{c}6.71 \\
(1.62,-3.12)\end{array}$ & $\begin{array}{c}3.20 \\
(0.98,5.43)\end{array}$ & $\begin{array}{c}1.92 \\
(1.23,2.61)\end{array}$ & $\begin{array}{l}443 \\
(30)\end{array}$ \\
\hline Uruguay & 0.68 & $\begin{array}{c}5.41 \\
(1.50,-3.35)\end{array}$ & $\begin{array}{c}1.91 \\
(0.58,3.23)\end{array}$ & $\begin{array}{c}1.86 \\
(1.19,2.52)\end{array}$ & $\begin{array}{l}443 \\
(30)\end{array}$ \\
\hline
\end{tabular}

\footnotetext{
*The first two columns record the estimates provided by Akgiray et al. The third column contains our estimates of the tail index on the basis of the same sample and using $m=18$. The last two columns rerecord estimates for extended samples, the sample size $n$ and number of order statistics $m$. The latter number was chosen on the basis of simulations. In parentheses are the 95 per cent confidence intervals (Akgiray et al. used twice the standard deviation instead of 1.96).
}

periods: Bretton Woods (the sample conforms to Westerfield, 1977; and Rogalski and Vinso, 1978, covering 1962.71); the first part of the float (the sample conforms to Boothe and Glassman, 1987, covering 1973-87); and the entire float (1973-91) for five different US dollar exchange rates. We first estimated tail indexes for the upper and lower index separately, but we could never reject symmetry (by the $Q$-test). Therefore, we decided to pool the extremes from both tails in order to increase the efficiency. In comparison with existing studies that commit to estimating a specific distribution model, the following can be noted.

The fixed regime Dmark, Pound and Canadian Dollar estimates are similar to Westerfield's (1977) stable estimates, but differ drastically from Rogalski and Vinso's (1978) Student- $t$ estimates as these hover around 3.7. In contrast, the estimate for the Guilder rate is above 2, which was not possible in Westerfield's study due to the restriction $1 \leqslant \beta<2$ in the estimation procedure. Nevertheless, the estimate is still significantly below the 3.9 value reported by Rogalski and Vinso. The other studies do not report estimates for the Yen. For the floating 
TABLE 3. Tail indexes for different exchange rate regimes.*

\begin{tabular}{lccccc}
\hline & $\alpha_{1}$ & $\alpha_{2}$ & $\alpha_{3}$ & & \\
& fix $(62-71)$ & float $(73-84)$ & float $(73-91)$ & $\alpha_{1}=\alpha_{2}$ & $\alpha_{1}=\alpha_{3}$ \\
\hline$n$ & 485 & 605 & 962 & & \\
$m$ & 51 & 54 & 81 & & \\
Dmark & 1.20 & 3.45 & 3.51 & $\neq$ & $\neq$ \\
Pound & $(0.86,1.52)$ & $(2.53,4.37)$ & $(2.75 .4 .28)$ & & $\neq$ \\
Yen & 1.14 & 3.21 & 3.58 & $\neq$ & $\neq$ \\
Guilder & $(0.82,1.45)$ & $(2.35,4.06)$ & $(2.80,4.36)$ & & \\
Can Dollar & 1.26 & 2.74 & 2.74 & $\neq$ & \\
& $(0.91-1.60)$ & $(2.01-3.47)$ & $(2.15-3.34)$ & & \\
& 2.42 & 3.35 & 3.45 & $2.26,3.25$ & $2.52,3.23$ \\
& $(1.75,3.08)$ & $(2.45,4.24)$ & $(2.70,4.21)$ & & \\
& 1.59 & 2.66 & 2.99 & $\neq$ & \\
\hline
\end{tabular}

* Estimates are based on end of the week recordings for the following periods: fixed exchange rates, January 4, 1962 to April 29, 1971 (source: Financial Times); first float, January 5, 1973 to February 6, 1987 (source: Harrisbank); second float, January 5, 1973 to June 14, 1991 (source: Harrisbank and Datastream). Point estimates are calculated by pooling the extremes from both tails. The 95 per cent asymptotic confidence intervals are given between brackets. The last two columns record the $Q$-test for equality of the tail indexes; the $\neq$ sign indicates rejection, otherwise the region of acceptance is recorded.

regime, the Dmark, Pound and Guilder estimates are in line with the Student- $t$ values of 3.9 reported by Rogalski and Vinso, and with the values obtained by Boothe and Glassman (1987) using daily data and the Student- $t$ model. Our estimates for the Canadian dollar are lower, while our estimates for the Yen are higher. These estimates are of course out of line with the stable estimates reported by Westerfield, and Boothe and Glassman, as these are necessarily below 2 .

Thus the advantages of our approach now come to the fore as it immediately detects the apparent model switch due to the regime change, whereas the other studies can and do not uncover this phenomenon. Comparing the two regimes we find the stable model is a tenable hypothesis for the period of fixed rates, while the Student- $t$ or ARCH model prevails during the period of the float. Note that the results of the $Q$-test reported in columns 4 and 5 show that the fix and floating regimes are significantly different from each other. The other studies cannot detect this because their estimates are conditional on specific nonnested distributions, while our estimation procedure admits the entire class of fat tailed distributions. The estimates for the float are both significantly greater than 2 in all cases (using a one-sided test of hypothesis), and above the point estimates for fixed rates.

These results are intuitively plausible for the following reason. Recall that the higher the tail index, the lower is the probability mass in the tails. This result thus substantiates the notion that flexible exchange rate regimes tend to smooth the movements in the foreign exchange markets. Given this conclusion for the fixed and free float regimes it is also of some interest to consider the halfway station of the EMS target zone. In the Appendix we briefly summarize the results which appeared in Koedijk et al. (1990), who report tail index estimates for EMS 
rates. They did find indexes below, but not too much different from, 2 for both the snake and the EMS period. Apparently, the snake and EMS arrangements are closer to the Bretton Woods setup than the (dirty) float of the US dollar rates. ${ }^{11}$ The benefits of the EMS may instead derive from changes in the center of the density. But this issue is addressed elsewhere and is outside the scope of the present methodology.

\section{Conclusions}

This paper addresses the issue of comparing the empirical unconditional distribution function of foreign exchange rate returns for different exchange rate regimes. In order to pursue this issue, one needs a statistical model which is robust across different specific alternatives. The reason is that an exchange rate regime switch may not only cause a change in parameters but also a change in the entire data-generating mechanism. Traditional modeling is precommitted to a specific nonnested maintained hypotheses. Therefore, different values for either the characteristic exponent or the degrees of freedom parameter under different exchange rate regimes cannot be directly ascribed to regime differences as this may stem from maintaining the wrong model. Extremal value theory offers a way to nest the different models. Regardless of the specific exchange rate regime in place, all exchange rate return d.f. exhibit the fat tail property. This stylized fact is exploited by extremal value theory. For application one needs to estimate the so-called tail index which is indicative of the amount of tail thickness, but conventional m.1. procedures are not always the best way to proceed in this area. In order to overcome the lack of precision associated with the m.l. procedure for estimating the tail shape, we proposed using the more efficient nonparametric I Iill estimator. Its uses and relative efficiency were demonstrated by a simulation study and on real data.

This estimator was then used to estimate the tail index for several different regimes: black markets, Bretton Woods, flexible exchange rates, the snake, and the EMS. Remarkably, all tail indexes are found to be in the same range between 1 and 2, except for the flexible exchange rate regime. The latter regime had significantly higher tail indexes. The economic interpretation of this result is that a float lets exchange rates adjust more smoothly than any other regime that involves some amount of fixity. Of course the other regimes may have had d.f.s with different center characteristics. But in some sense the extreme movements count the most for the economic process, and here Friedman's (1953) prediction about the workings of flexible exchange rates seems to be borne out.

\section{Appendix EMS rates}

This appendix provides a summary of the results for the EMS and the snake as reported in Koedijk et al. (1990). 
TABLE 4. Tail indexes for EMS exchange rates.*

\begin{tabular}{|c|c|c|c|}
\hline & Snake & EMS & \\
\hline$n$ & 412 & 412 & \\
\hline$m$ & 28 & 28 & \\
\hline French Franc/Dmark & $\begin{array}{c}1.92 \\
(1.21,2.63)\end{array}$ & $\begin{array}{c}1.25 \\
(0.78,1.71)\end{array}$ & $1.07,1.82$ \\
\hline Italian Lire/Dmark & $\begin{array}{c}1.41 \\
(0.89,1.93)\end{array}$ & $\begin{array}{c}1.78 \\
(1.12,2.44)\end{array}$ & $1.07,2.03$ \\
\hline Dutch Guilder/Dmark & $\begin{array}{c}1.69 \\
(1.06,2.32)\end{array}$ & $\begin{array}{c}1.62 \\
(1.02,2.22)\end{array}$ & $1.11,2.19$ \\
\hline Pound/Dmark & $\begin{array}{c}2.66 \\
(1.67-3.64)\end{array}$ & $\begin{array}{c}2.17 \\
(1.37-2.98)\end{array}$ & $1.63-3.11$ \\
\hline
\end{tabular}

*Estimates are based on end of the week recordings for the following periods: Snake, April 1971 to March 1979; EMS, March 1979 to March 1987.

\section{Notes}

1. An excellent review of the field is contained in Boothe and Glassman (1987). Other models used to capture the leptokurtic return distribution are the discrete mixture of normals; the mixed diffusion jump process (see, e.g., Akgiray and Booth, 1988; and Tucker and Pond, 1988); and the power exponential or GED (see Hsieh, 1989). Among the non i.d.d. processes the ARMA and ARCH models are most popular. The ARCH process is especially relevant since it exhibits clusters of volatility, which is the other well known empirical property of exchange rate returns ( see, e.g., Diebold, 1988; Hsieh, 1989; and De Vries, 1991).

2. The study by Farber $e$ al. (1977) is omitted as their division of periods is now questionable given the currently available data.

3. Their study contains an application of extremal analysis to EMS issues. Here we broaden the scope and investigate several different exchange rate regimes.

4. Triangular arbitrage implies that returns on different currencies are linked. This places additive restrictions on the distributions for the different returns. In fact, this explains the appeal of the stable model, as these are the only distributions which are invariant under addition. In the case of black markets, triangular arbitrage may be absent or imperfect, and hence the tail indices may be different for different exchange rate return series.

5. The normal mixtures of the normal, mixed diffusion jump processes and the power exponential are all thin tailed instead of fat tailed so that $\alpha=\infty$ (see Theorem 1.6.1 in Leadbetter et al., 1983).

6. This procedure for estimating $\alpha$ must not be confused with the m.l. procedure for fitting a specific d.f. in order to estimate all parameters of this d.f.

7. We also conducted an experiment with the much larger sample size $n=6000$. As the results of this experiment were quite similar to those for the smaller sample, this experiment is not recorded here, but is available from the authors upon request.

8. For Columbia and Chile our data set differs slightly from the data set in Akgiray et al. to correct for the monetary reform in Chile and some recording differences. All data are available from the second author upon request. The Akgiray et al. data cover the period April 1973 to April 1983, while the extended data set runs from 1947 to 1983, except for the data for Bolivia, Chile, and Costa Rica which start in 1948, the data for Columbia and Paraguay which start in 1952, and the data for El Salvador which start in 1961. The exchange rate data are monthly quotations of black market rates from various issues of Pick's Currency Yearbook.

9. The right endpoints of the confidence intervals were computed on the basis of the information given by Akgiray et al. in their Table 3. 
10. In the computations, we used $m$-levels on the basis of the simulations of Table 1 and simulations for the extended sample sizes (not summarized here). The simulations show that the optimal $m$-level varies with the $x$ values, and the latter are of course unknown. In general, we decided to trade off unbiasedness against efficiency, cf. Table 1 , and used the number of order statistics suggested by the Cauchy simulations.

11. As was pointed out to us by a referee, triangular arbitrage relates the Guilder/Dmark rate tail estimate of Table 4 to the estimates for the Dmark/US and Guilder/US rates of Table 3, cf. note 4. Suppose that the latter two exchange rate returns are independent, then by Theorem 2 of Koedijk et al. (1990) the Guilder/Dmark rate should have a tail index of the same size as the other two rates. The value of the Guilder/Dmark ratc, though, is considerably lower. This may be an interesting problem to follow up in future research. Also note that for the Pound, which effectively did not participate in the EMS, the problem seems absent.

\section{References}

Akgiray, V., G.G. Booth, and B. Sfiffrt, 'Distribution Properties of Latin American Black Market Exchange Rates,' Journal of International Money' and Finance, March 1988, 7: $37-48$.

Akgiray, V., and G.G. Booth, 'Mixed Diffusion-Jump Process Modeling of Exchange Rate Movements,' Review of Economics and Statistics, 1988, 70: $631-637$.

Boothe. P., and D. Glassman, 'The Statistical Distribution of Exchange Rates: Empirical Evidence and Economic Implications,' Journal of International Economics, May 1987, 23: $297-320$.

Diebold, F.X., Empirical Modeling of Exchange Rate Dynamics, New York: Springer-Verlag. 1988.

Dumolchel, W.H., 'Estimating the Stable Index $\alpha$ in Order to Measure Tail Thickness: A Critique," Annals of Statistics, December 1983, 11: 1019 1031.

Farbir, A., R. Roll, and B. Solnik. 'An Empirical Study of Risk Under Fixed and Flexible Exchange," in K. Brunner and A.H. Meltzer, eds, Stabilization of the Domestic and International Fconomy, Carnegie-Rochester Conference Series on Public Policy, 1977, $235-266$.

Frifidman, M., 'The Case for Flexible Exchange Rates, Essays in Positite Economics, Chicago: University of Chicago Press, 1053, 157203.

Goldie, C.M., AND R.L. Smith, 'Slow Variation with Remainder: Theory and Applications, Quarterly Joumal of Mathematics, Oxford 2nd series, 1987, $45 \cdots 71$.

Han. L., de. S.I. Resnick. H. Rootzen, and C.G. de Vrifs. 'Extremal Behaviour of Solutions to a Stochastic Difference Equation with Applications to ARCH-Processes,' Stochastic Processes and their Applications, 1989, $213-224$.

HiLl, B.M., 'A Simple General Approach to Inference about the Tail of a Distribution.' Annals of Statistics, 1975, 3: 11631173.

Hols, M.C.A.B., and C.G. dr VRIES. 'The Limiting Distribution of Extremal Exchange Rate Returns,' Journal of Applied Econometrics, 1991, 6: 287-302.

Hsifr, D.A., 'Modeling Heteroscedasticity in Daily Foreign-Exchange Rates,' Journal of Business and Economic Statistics, 1989, 7: 307-317.

Kofdij, K.G.. M.M.A. Schargans, and C.G. DF Vrifs, 'The Tail Index of Exchange Rate Returns,' Joumal of International Economics, August 1990, 29: 93108.

Leadbetter, M.R., G. Liniggren, and H. Rootzen, Exiremes and Related Properties of Random Sequences and Processes, New York: Springer-Verlag, 1983.

Mood, A.M., T.A. Graybill, and D.C. Boes, Introduction to the Theory of Statistics, New York: McGraw-Hill, 1974.

Rana, P.B., 'Fixed Versus Flexible Exchange Rates and Measurement of Exchange Rate Instability: Comment,' Journal of International Economics, 1984, 20: 307 - 310.

Rogalski, R.J.. anl J.D. Vi^so, 'Empirical Properties of Foreign Exchange Rates,' Journal of International Business Studies, Fall 1978, 9: 6979.

Sмiтн, R.H., 'Estimating Tails of Probability Distributions,' Annals of Statistics, 1987, 15: $1174-1207$. 
Tucker, A.L., And L. Pond, 'The Probability Distribution of Foreign Exchange Price Changes: Tests of Candidate Processes,' Review of Economic's and Statistics, 1988, 70: 638-647.

VRIEs, C.G. DF, 'On the Relation Between GARCH and Stable Processes,' Journal of Econometrics, June 1991, 51: 313-324.

Westerfield, J.M., 'An Examination of Foreign Exchange Risk Under Fixed and Floating Rate Regimes,' Journal of International Fronomics, May 1977, 7: 181-200. 